J.B.METZLER 


\section{Metzler Lexikon Weltliteratur}

1000 Autoren von der Antike bis zur Gegenwart

Band 1

A - F

Herausgegeben von Axel Ruckaberle

Verlag J. B. Metzler

Stuttgart - Weimar 


\section{Der Herausgeber}

Axel Ruckaberle ist Redakteur bei der Zeitschrift für Literatur "TEXT + KRITIK«, beim "Kritischen Lexikon zur deutschsprachigen Gegenwartsliteratur « (KLG) und beim »Kritischen Lexikon zur fremdsprachigen Gegenwartsliteratur « (KLfG).

Rund die Hälfte der in diesen Bänden versammelten Autorenporträts stammen aus den folgenden Lexika: "Metzler Lexikon englischsprachiger Autorinnen und Autoren «, herausgegeben von Eberhard Kreutzer und Ansgar Nünning, 2002/2006.

"Metzler Autoren Lexikon ", herausgegeben von Bernd Lutz und Benedikt Jeßing, 3. Auflage 2004.

"Metzler Lexikon amerikanischer Autoren «, herausgegeben von Bernd Engler und Kurt Müller, 2000.

"Metzler Autorinnen Lexikon «, herausgegeben von

Ute Hechtfischer, Renate Hof, Inge Stephan und Flora Veit-Wild, 1998.

"Metzler Lexikon antiker Autoren «, herausgegeben von Oliver Schütze, 1997.

In diesen Lexika befinden sich auch weiterführende Literaturhinweise zu jedem der Artikel.
Bibliografische Information Der Deutschen Nationalbibliothek

Die Deutsche Nationalbibliothek verzeichnet diese Publikation in der Deutschen Nationalbibliografie; detaillierte bibliografische Daten sind im Internet über $<$ http://dnb.d-nb.de> abrufbar.

ISBN-13: 978-3-476-02093-2

\section{ISBN 978-3-476-02094-9 ISBN 978-3-476-00127-6 (eBook) DOI 10.1007/978-3-476-00127-6}

Dieses Werk einschließlich aller seiner Teile ist urheberrechtlich geschützt. Jede Verwertung außerhalb der engen Grenzen des Urheberrechtsgesetzes ist ohne Zustimmung des Verlages unzulässig und strafbar. Das gilt insbesondere für Vervielfältigungen, Übersetzungen, Mikroverfilmungen und die Einspeicherung und Verarbeitung in elektronischen Systemen.

\section{C을 2006 Springer-Verlag GmbH Deutschland}

Ursprünglich erschienen bei J. B. Metzler'sche Verlagsbuchhandlung und Carl Ernst Poeschel Verlag GmbH in Stuttgart 2006

www.metzlerverlag.de

info@metzlerverlag.de 


\section{Vorwort}

Das Metzler Lexikon Weltliteratur versammelt mehr als 1000 Porträts von Schriftstellerinnen und Schriftstellern aus aller Welt sowie einige Artikel über anonym erschienene Einzelwerke - vom Beginn literarischen Schreibens bis in die Gegenwart. Ausgewählt wurden einerseits klassische, zum Kanon der Weltliteratur zählende Autorinnen und Autoren, andererseits Schriftsteller, vor allem der Gegenwartsliteraturen, die für deutschsprachige Leser/innen durch ihre Verbreitung im deutschen Sprachraum von Interesse sind. Daß eine solche Auswahl nicht die Weltliteratur insgesamt repräsentieren und nicht allen Vorstellungen von ihr gerecht werden kann, versteht sich von selbst.

Das Werk basiert auf den Metzler Lexika Amerikanische Autoren (2000) und Englischsprachige Autorinnen und Autoren (2002), dem Metzler Autoren Lexikon. Deutschsprachige Dichter und Schriftsteller vom Mittelalter bis zur Gegenwart ( $\left.{ }^{3} 2004\right)$, dem Metzler Autorinnen Lexikon (1998) und dem Metzler Lexikon antiker Autoren (1997), aus denen etwa die Hälfte der hier versammelten Artikel stammt. Den Herausgeber/innen der genannten Lexika, Bernd Engler und Kurt Müller, Eberhard Kreutzer und Ansgar Nünning, Bernd Lutz und Benedikt Jeßing, Oliver Schütze, Ute Hechtfischer, Renate Hof, Inge Stephan und Flora Veit-Wild sowie den Verfasserinnen und Verfassern der übernommenen Artikel sei herzlich gedankt.

Herzlich danke ich auch allen Verfasserinnen und Verfassern der neu erarbeiteten Beiträge, ganz besonders denjenigen, die bei der Auswahl der Autoren beratend mitgewirkt haben. Mein besonderer Dank gilt Ute Hechtfischer vom Metzler Verlag und Heinz Ludwig Arnold in Göttingen. Andrea Knigge danke ich von Herzen für ihre Unterstützung bei der Bearbeitung der neuen Beiträge und der Arbeit am Lexikon insgesamt.

Göttingen, im Juni 2006

Axel Ruckaberle
Bei der Schreibweise von Autornamen wurden statt wissenschaftlicher Umschriften zum Teil allgemein gebräuchliche Formen verwendet. In Fällen, bei denen die Abweichung beider Formen eine veränderte Stellung im Alphabet bedingt, werden Verweise gegeben, ebenso in Fällen, in denen heute gebräuchliche Formen von früheren Schreibweisen abweichen.

Ortsangaben und Nennungen von Herkunftsländern folgen nicht in allen Fällen historischen Gegebenheiten, sondern verweisen zum einfacheren Verständnis gelegentlich auf heutige Zugehörigkeiten. 


\section{Inhalt}

\section{Band 1:}

Vorwort V

Verzeichnis der Artikel IX

Mitarbeiterinnen und Mitarbeiter XXI

Artikel A-F 1-497

\section{Band 2:}

Artikel G-M 1-507

\section{Band 3:}

Artikel N-Z $\quad 1-483$ 


\section{Verzeichnis der Artikel}

A

Abe Kōbō

Abélard, Pierre

Abramov, Fëdor

Abū l-Farağ al-Işfahānī

Achebe, Chinua

Achmatova, Anna

Ackroyd, Peter

Adonis

Ady, Endre

Aesop

Ağaoğlu, Adalet

Agnon, Samuel Josef

Ai Qing

Aichinger, Ilse

Aidoo, Ama Ata

Aischylos

Aizpuriete, Amanda

Ajtmatov, Cingiz

Aksënov, Vasilij

Akutagawa Ryūnosuke

Alain-Fournier, Henri

Alavi, Bozorg

Albee, Edward

Alexanderroman

Alexandrou, Aris

Ali, Sabahattin

Allende, Isabel

Almada Negreiros, José Sobral de

Almqvist, Carl Jonas Love

Altägyptisches Totenbuch

Amado, Jorge

Amichai, Jehuda

Amis, Kingsley

Amis, Martin

Anakreon

Anand, Mulk Raj

Andersch, Alfred

Andersen, Hans Christian

Andersen Nexø, Martin

Anderson, Sherwood

Andrade, Carlos Drummond de

Andrić, Ivo

Andrzejewski, Jerzy

Anouilh, Jean

Antimachos von Kolophon

Apollinaire, Guillaume

Appelfeld, Aharon
Aragon, Louis

Archilochos

Arden, John

Arenas, Reinaldo

Arendt, Erich

Aretino, Pietro

Arghezi, Tudor

Ariost, Ludovico

Aristophanes

Aristoteles

Armah, Ayi Kwei

Arnim, Achim von

Arnim, Bettine von

Arrabal, Fernando

Artaud, Antonin

Artmann, H.C.

Ashbery, John

Assis, Joaquim Maria Machado de

Asturias, Miguel Ángel

'Atțār

Atwood, Margaret

Aub, Max

Auden, W.H.

Augustinus

Austen, Jane

Auster, Paul

Ayckbourn, Alan

\section{B}

Bâ, Amadou Hampâté

Bâ, Mariama

Ba Jin

Babel', Isaak

Bachmann, Ingeborg

Bacon, Francis

Baldwin, James

Balzac, Honoré de

Bang, Herman

Banville, John

Baratynskij, Evgenij

Barnes, Djuna

Baroja y Nessi, Pío

Barth, John

Barthelme, Donald

Barthes, Roland

Bashō

Bassani, Giorgio

Bataille, Georges 
Baudelaire, Charles

Beaumarchais, Pierre Augustin Caron de

Beauvoir, Simone de

Beckett, Samuel

Beckford, William

Bécquer, Gustavo Adolfo

Behan, Brendan

Behn, Aphra

Bei Dao

Belli, Gioconda

Bellman, Carl Michael

Bello, Andrés

Bellow, Saul

Belyj, Andrej

Ben Jelloun, Tahar

Benet, Juan

Benn, Gottfried

Beowulf

Berger, John

Bernanos, Georges

Bernhard, Thomas

Bessa-Luis, Augustina

Beti, Mongo

Bhagavadgitā

Bialik, Chaim Nachman

Bibel

Bioy Casares, Adolfo

Bishop, Elizabeth

Bitov, Andrej

Blake, William

Blaman, Anna

Blandiana, Ana

Blasco Ibáñez, Vicente

Blixen, Karen

Blok, Aleksandr

Blyton, Enid

Boal, Augusto

Bobrowski, Johannes

Boccaccio, Giovanni

Boileau, Nicolas

Bolaño, Roberto

Böll, Heinrich

Bond, Edward

Bonnefoy, Yves

Bontempelli, Massimo

Boon, Louis Paul

Borges, Jorge Luis

Boudjedra, Rachid

Bowles, Paul

Boye, Karin

Bradford, William

Brandys, Kazimierz
Brant, Sebastian

Braun, Volker

Brautigan, Richard

Brecht, Bertolt

Brentano, Clemens

Breton, André

Breytenbach, Breyten

Brink, André

Brinkmann, Rolf Dieter

Broch, Hermann

Brodskij, Iosif

Brontë, Charlotte

Brontë, Emily

Browning, Robert

Bryant, William Cullen

Buch der Lieder

Buch der Wandlungen

Büchner, Georg

Bulgakov, Michail

Bunin, Ivan

Buonarotti, Michelangelo

Burgess, Anthony

Burns, Robert

Burroughs, William

Busch, Wilhelm

Butler, Samuel

Butor, Michel

Buzzati, Dino

Byatt, A.S.

Byron, George Gordon Noël Lord

C

Cabral de Melo Neto, João

Cabrera Infante, Guillermo

Calderón de la Barca, Pedro

Calvino, Italo

Camões, Luís Vaz de

Camus, Albert

Canetti, Elias

Cankar, Ivan

Cantar de Mío Cid

Canth, Minna

Čapek, Karel

Capote, Truman

Cardenal, Ernesto

Carey, Peter

Carlyle, Thomas

Carmina Burana

Carpelan, Bo

Carpentier, Alejo

Carroll, Lewis

Carter, Angela 
Carver, Raymond

Casanova, Giovanni Giacomo

Castelo Branco, Camilo

Castiglione, Baldassare

Catull

Cayrol, Jean

Čechov, Anton

Cela, Camilo José

Celan, Paul

Celestina $\rightarrow$ Rojas, Fernando de

Céline, Louis-Ferdinand

Cendrars, Blaise

Cernuda, Luis

Cervantes, Miguel de

Césaire, Aimé

Chajjām, Omar

Chamisso, Adelbert von

Chandler, Raymond

Chanson de Roland

Char, René

Charrat, Edwar al- $\rightarrow$ Kharrat, Edwar al-

Charms, Daniil

Chateaubriand, François-René

Chatwin, Bruce

Chatzis, Dimitris

Chaucer, Geoffrey

Chen Ruoxi

Cheney-Coker, Syl

Chirbes, Rafael

Chlebnikov, Velemir

Chodasevič, Vladislav

Choderlos de Laclos, Pierre Ambroise François

Chrestien de Troyes

Christensen, Inger

Christie, Agatha

Churchill, Caryl

Christine de Pizan

Cicero

Cid $\rightarrow$ Cantar de Mío Cid

Cioran, Emile Michel

Cixous, Hélène

Clarín

Claudel, Paul

Claus, Hugo

Clemens, Samuel Langhorne $\rightarrow$ Mark Twain

Cocteau, Jean

Coetzee, J.M.

Coleridge, Samuel Taylor

Colette

Condé, Maryse

Congreve, William

Conrad, Joseph
Constant, Benjamin

Cooper, James Fenimore

Coover, Robert

Corneille, Pierre

Cortázar, Julio

Cosić, Bora

Couto, Mia

Crane, Stephen

Creeley, Robert

Cruz, Sor Juana Inés de la

Cummings, E.E.

Cvetaeva, Marina

D

Dadié, Bernard B.

Dağlarca, Fazıl Hüsnü

Daif, Rashid al-

D’Annunzio, Gabriele

Dante Alighieri

Darío, Rubén

Darwish, Mahmud

Daudet, Alphonse

Defoe, Daniel

Deledda, Grazia

Delibes, Miguel

DeLillo, Don

Déry, Tibor

Deržavin, Gavrila

Desai, Anita

Devi, Mahasweta

Dib, Mohammed

Dickens, Charles

Dickinson, Emily

Diderot, Denis

Didion, Joan

Dinescu, Mircea

Ding Ling

Diwan-Poesie

Djebar, Assia

Djilas, Milovan

Döblin, Alfred

Doctorow, E.L.

Doderer, Heimito von

Donne, John

Dos Passos, John

Dostoevskij, Fedor

Doulatābādi, Mahmud

Dove, Rita

Doyle, Arthur Conan

Drieu la Rochelle, Pierre

Droste-Hülshoff, Annette von

Dryden, John 
Dschāmi, Moulānā Nuroddin Abdorrahmān

Du Fu

Dumas, Alexandre (fils)

Dumas, Alexandre (père)

Duras, Marguerite

Durrell, Lawrence

Dürrenmatt, Friedrich

\section{E}

Ebner-Eschenbach, Marie von

Eça de Queirós, José Maria

Eco, Umberto

Edda

Ehrenburg, Il'ja

Eich, Günter

Eichendorff, Joseph von

Ekelöf, Gunnar

Ekman, Kerstin

Eliade, Mircea

Eliot, George

Eliot, T.S.

Ellison, Ralph

Éluard, Paul

Elytis, Odysseas

Emecheta, Buchi

Emerson, Ralph Waldo

Eminescu, Mihai

Enchi, Fumiko

Endō Shusaku

Enquist, Per Olov

Enzensberger, Hans Magnus

Erasmus von Rotterdam

Erdrich, Louise

Ernaux, Annie

Esterházy, Péter

Euripides

Evans, Mary Ann $\rightarrow$ Eliot, George

Evtušenko, Evgenij

\section{F}

Faik, Sait

Fallada, Hans

Farah, Nuruddin

Farrohzãd, Forūg

Faulkner, William

Federman, Raymond

Ferdousi

Fernández de Lizardi, José Joaquín

Ferré, Rosario

Ferreira, António

Feuchtwanger, Lion

Fichte, Hubert
Fielding, Henry

Fitzgerald, F. Scott

Firdausi $\rightarrow$ Ferdousi

Flaubert, Gustave

Fleißer, Marieluise

Fo, Dario

Fonseca, José Rubem

Fontane, Theodor

Ford, Richard

Forster, E.M.

Fowles, John

Frame, Janet

France, Anatole

Fried, Erich

Frisch, Max

Frost, Robert

Fuentes, Carlos

Fugard, Athol

G

Gadda, Carlo Emilio

Gaddis, William

Gālib, Mirzā Asadullāh Khan

Galsworthy, John

Gao Xingjian

García Lorca, Federico

Garcia Márquez, Gabriel

Garrett, João Baptista da Silva

Gary, Romain

Gautier, Théophile

Genet, Jean

George, Stefan

Gezelle, Guido

Ghitani, Gamal al-

Ghosh, Amitav

Gibran, Khalil

Gide, André

Gilgamesch-Epos

Ginsberg, Allen

Ginzburg, Natalia

Giono, Jean

Giraudoux, Jean

Glissant, Édouard

Goethe, Johann Wolfgang

Gogol', Nikolaj

Golding, William

Goldoni, Carlo

Goldsmith, Oliver

Golširi, Hušang

Gombrowicz, Witold

Gómez de la Serna, Ramón 
Gončarov, Ivan

Goncourt, Edmond u. Jules de

Góngora y Argote, Don Luis de

Gordimer, Nadine

Gorgāni, Fahrod-din Ascad

Gor'kij, Maksim

Gottfried von Straßburg

Gotthelf, Jeremias

Goytisolo, Juan

Grabbe, Christian Dietrich

Gracián

Gracq, Julien

Granin, Daniil

Grass, Günter

Gray, Thomas

Green, Julien

Greene, Graham

Grillparzer, Franz

Grimm, Jakob u. Wilhelm

Grimmelshausen, Hans Jacob Christoph von

Grünbein, Durs

Gryphius, Andreas

Guarini, Giovanni Battista

Guillén, Jorge

Günderrode, Karoline von

Gunesekera, Romesh

Gunnarsson, Gunnar

Guo Moruo

Gurnah, Abdulrazak

Gustafsson, Lars

\section{H}

Haasse, Hella S.

Haavikko, Paavo

Habibi, Emil

Hafis

Hallgrímsson, Jónas

Hammett, Dashiell

Hamsun, Knut

Handke, Peter

Hardy, Thomas

Harīī, Abū Muhammad al-

Hart, Maarten 't

Hartmann von Aue

Hašek, Jaroslav

Hauff, Wilhelm

Hauptmann, Gerhart

Havel, Václav

Hawthorne, Nathaniel

Hayyām, 'Omar $\rightarrow$ Chajjām, Omar

Head, Bessie

Heaney, Seamus
Hebbel, Christian Friedrich

Hebel, Johann Peter

Hébert, Anne

Hedāyat, Șādeq

Heijden, A.F.Th. van der

Hein, Christoph

Heine, Heinrich

Heißenbüttel, Helmut

Hemingway, Ernest

Herbert, George

Herbert, Zbigniew

Herculano, Alexandre

Herder, Johann Gottfried

Hermans, Willem Frederik

Herwegh, Georg

Herzberg, Judith

Hesiod

Hesse, Hermann

Heym, Georg

Heyse, Paul

Highsmith, Patricia

Hikmet, Nâzım

Hildebrandslied

Hłasko, Marek

Hochhuth, Rolf

Høeg, Peter

Hoffmann, E.T.A.

Hoffmann von Fallersleben, August Heinrich

Hofmannsthal, Hugo von

Holberg, Ludvig

Hölderlin, Friedrich

Homer

Honglou meng $\rightarrow$ Traum der roten Kammer

Hooft, Pieter Corneliszoon

Hopkins, Gerard Manley

Horaz

Horváth, Ödön von

Houellebecq, Michel

Hrabal, Bohumil

Hughes, Langston

Hughes, Ted

Hugo, Victor

Hulme, Keri

Huxley, Aldous

Huysmans, Joris-Karl

I

$I$ Ging $\rightarrow$ Buch der Wandlungen

Ibn al-'Arabì

Ibn Hazm, 'Ali

Ibsen, Henrik

Ikstena, Nora 
Inoue Yasushi

Ionesco, Eugène

Irving, John

Irving, Washington

Iskander, Fazil'

Ivanauskaitè, Jurga

\section{J}

Jacobsen, Jens Peter

Jahnn, Hans Henny

James, Henry

Jandl, Ernst

Jansson, Tove

Jarry, Alfred

Jarunková, Klára

Jean Paul

Jehoschua, Abraham B.

Jelinek, Elfriede

Jensen, Johannes Vilhelm

Jiménez, Juan Ramón

Johnson, Eyvind

Johnson, Samuel

Johnson, Uwe

Jonson, Ben

Jorge, Lídia

José, Francisco Sionil

Joyce, James

József, Attila

Jünger, Ernst

\section{$\mathbf{K}$}

Kabbala

Kadare, Ismail

Kafka, Franz

Kalidasa

Kamasutra

Kanafani, Ghassan

Kane, Sarah

Kaniuk, Yoram

Karamzin, Nikolaj

Karvaš, Peter

Kaschnitz, Marie Luise

Kästner, Erich

Kateb, Yacine

Kavafis, Konstantinos

Kawabata Yasunari

Kazantzakis, Nikos

Keats, John

Keller, Gottfried

Kemal, Orhan

Kemal, Yaşar

Kempowski, Walter
Kerouac, Jack

Kertész, Imre

Khalifa, Sahar

Kharrat, Edwar al-

Khoury, Elias

Kielland, Alexander Lange

Kierkegaard, Søren

King, Stephen

Kipling, Rudyard

Kirsch, Sarah

Kiš, Danilo

Kivi, Aleksis

Kleist, Heinrich von

Klíma, Ivan

Kloos, Willem Johannes Theodorus

Klopstock, Friedrich Gottlieb

Kochanowski, Jan

Koeppen, Wolfgang

Kohout, Pavel

Koidula, Lydia

Kolář, Jiří

Kolmar, Gertrud

Koni, Ibrahim al-

Kono Taeko

Konwicki, Tadeusz

Kopfkissenbuch $\rightarrow$ Sei Shōnagon

Koran

Kosztolányi, Dezső

Kourouma, Ahmadou

Krall, Hanna

Kraus, Karl

Kristof, Agota

Krleža, Miroslav

Krolow, Karl

Kronauer, Brigitte

Kross, Jaan

Kunčinas, Jurgis

Kundera, Milan

Kunert, Günter

Kuśniewicz, Andrzej

$\mathbf{L}$

La Fontaine, Jean de

La Rochefoucauld, François

Laclos, Choderlos de $\rightarrow$ Choderlos de Laclos

Lagerkvist, Pär

Lagerlöf, Selma

Landolfi, Tommaso

Langgässer, Elisabeth

Lao She

Larkin, Philip

Lasker-Schüler, Else 
Lavant, Christine

Lawrence, D.H.

Laxness, Halldór

le Carré, John

Le Clézio, J.M.G.

Lec, Stanisław Jerzy

Leino, Eino

Leiris, Michel

Lem, Stanisław

Lenz, Hermann

Lenz, Jakob Michael Reinhold

Lenz, Siegfried

Leopardi, Giacomo

Lermontov, Michail

Leskov, Nikolaj

Lessing, Gotthold Ephraim

Lessing, Doris

Levi, Carlo

Levi, Primo

Lezama Lima, José

Lidman, Sara

Liksom, Rosa

Lindgren, Astrid

Linhartová, Věra

Linna, Väinö

Lispector, Clarice

Llamazares, Julio

Lobo Antunes, António

Lohenstein, Daniel Caspar von

Lomonosov, Michail

London, Jack

Lönnrot, Elias

Loti, Pierre

Lowry, Malcolm

Lu Xun

Lubis, Mochtar

Lucebert

Luik, Viivi

Lukian

Lukrez

Luther, Martin

M

Maalouf, Amin

Ma'arri, Abū l-Aläa’al-

Machado y Ruiz, Antonio

Machfus, Nagib

Machiavelli, Niccolò

Maeterlinck, Maurice

Mahābhārata

Mailer, Norman

Majakovskij, Vladimir
Malamud, Bernard

Malaparte, Curzio

Mallarmé, Stéphane

Malouf, David

Malraux, André

Mandel'štam, Osip

Mándy, Iván

Mankell, Henning

Mann, Heinrich

Mann, Klaus

Mann, Thomas

Mansfield, Katherine

Manzoni, Alessandro

Márai, Sándor

Maraini, Dacia

Marechera, Dambudzo

Marías, Javier

Marinetti, Filippo Tommaso

Marivaux, Pierre Carlet de

Mark Aurel

Mark Twain

Marlowe, Christopher

Maroufi, Abbas

Marsé, Juan

Martin du Gard, Roger

Maugham, W. Somerset

Maupassant, Guy de

Mauriac, François

May, Karl

Mayröcker, Friederike

McCullers, Carson

McEwan, Ian

Melo, Francisco Manuel de

Melville, Herman

Memmi, Albert

Menander

Meri, Veijo

Mérimée, Prosper

Mertens, Pierre

Mészöly, Miklós

Meyer, Conrad Ferdinand

Michaux, Henri

Mickiewicz, Adam

Middleton, Thomas

Miller, Arthur

Miller, Henry

Miłoz, Czesław

Milton, John

Mishima Yukio

Mistral, Frédéric

Mistral, Gabriela

Mňačko, Ladislav 
Mo Yan

Mochtar Lubis $\rightarrow$ Lubis, Mochtar

Molière

Molina, Tirso de $\rightarrow$ Tirso de Molina

Molnár, Ferenc

Montaigne, Michel de

Montale, Eugenio

Montero, Rosa

Montesquieu, Charles-Louis de

Montherlant, Henry de

Moor, Margriet de

Morante, Elsa

Moravia, Alberto

Morgenstern, Christian

Morgner, Irmtraud

Mori Ōgai

Mörike, Eduard

Moritz, Karl Philipp

Morrison, Toni

Mrożek, Slawomir

Mưallaqāa

Mudimbe, V.Y.

Mulisch, Harry

Müller, Heiner

Müller, Herta

Multatuli

Munif, Abdalrahman

Muñoz Molina, Antonio

Munro, Alice

Murakami Haruki

Murasaki Shikibu

Murdoch, Iris

Murray, Les

Musil, Robert

Musset, Alfred de

Mutafčieva, Vera

Mwangi, Meja

N

Nabokov, Vladimir

Nádas, Péter

Naipaul, V.S.

Narayan, R.K.

Natsume Sōseki

Naum, Gellu

Neidhart

Němcová, Božena

Németh, László

Neruda, Jan

Neruda, Pablo

Nerval, Gérard de

Nesin, Aziz
Nestroy, Johann Nepomuk

Nezāami, Abū Mohammad $\rightarrow$ Nișami, Abu Mohammad

Nganang, Patrice

Ngugi wa Thiongo, James

Nibelungenlied

Nisami, Abu Moḥammad

Nooteboom, Cees

Novalis

Nurowska, Maria

O

Oates, Joyce Carol

Ocampo, Silvina

O'Casey, Sean

O'Connor, Flannery

Ōe Kenzaburō

Oehlenschläger, Adam

Ondaatje, Michael

O'Neill, Eugene

Onetti, Juan Carlos

Ortega y Gasset, José

Ortese, Anna Maria

Orwell, George

Osborne, John

Ostrovskij, Aleksandr

Ouologuem, Yambo

Ovid

Oz, Amos

\section{$\mathbf{P}$}

Paemel, Monika van

Pagnol, Marcel

Palamas, Kostis

Pamuk, Orhan

Pascal, Blaise

Pascoli, Giovanni

Pasolini, Pier Paolo

Pasternak, Boris

Paustovskij, Konstantin

Pavese, Cesare

$\mathrm{Paz}$, Octavio

Pelevin, Viktor

Pepetela

Pepys, Samuel

Perec, Georges

Pérez Galdós, Benito

Peri Rossi, Cristina

Pessoa, Fernando

Petőfi, Sándor

Petrarca, Francesco

Petron 
Petruševskaja, Ljudmila

Pétursson, Hallgrímur

Phillips, Caryl

Pilnjak, Boris

Pindar

Pinget, Robert

Pinter, Harold

Pirandello, Luigi

Plath, Sylvia

Platon

Platonov, Andrej

Plautus

Poe, Edgar Allan

Pope, Alexander

Pound, Ezra

Premcand

Prešeren, France

Prévert, Jacques

Priestley, J.B.

Proust, Marcel

Puig, Manuel

Puškin, Aleksandr

Pynchon, Thomas

$\mathbf{Q}$

Queneau, Raymond

Queirós, José Maria Eça $\rightarrow$ Eça de Queirós, José Maria

Quevedo, Francisco de

$\mathbf{R}$

Raabe, Wilhelm

Rabelais, François

Racine, Jean

Rāmãyana

Rao, Raja

Räuber vom Liang Schan Moor

Remarque, Erich Maria

Remizov, Aleksej

Rendra

Reuter, Fritz

Reuter, Bjarne

Reve, Gerard

Rhys, Jean

Richardson, Samuel

Rilke, Rainer Maria

Rimbaud, Jean Arthur

Ringelnatz, Joachim

Rinser, Luise

Ritsos, Jannis

Roa Bastos, Augusto

Robbe-Grille, Alain
Rodoreda, Mercé

Roidis, Emmanouil

Rojas, Fernando de

Rolandslied $\rightarrow$ Chanson de Roland

Rolland, Romain

Ronsard, Pierre de

Rosa, João Guimarães

Roth, Joseph

Roth, Philip

Rousseau, Jean-Jacques

Rowling, J.K.

Roy, Arundhati

Różewicz, Tadeusz

Rühmkorf, Peter

Rulfo, Juan

Rumi

Rushdie, Salman

Rybakov, Anatolij

S

Sa adāwī, Nawāl as-

Saadi

Saarikoski, Pentti

Sábato, Ernesto

Sachs, Hans

Sachs, Nelly

Sade, Donatien Alphonse François Marquis de

Sainte-Beuve, Charles-Augustin de

Saint-Exupéry, Antoine de

Saint-John Perse

Salih, Tayyib

Salinger, J.D.

Sallust

Šāmlu, Ahmad $\rightarrow$ Schāmlu, Ahmad

Sammān, Gāàa as-

Sand, George

Sappho

Saramago, José

Saro-Wiwa, Ken

Sarraute, Nathalie

Sartre, Jean-Paul

Schädlich, Hans Joachim

Schāmlu, Ahmad

Schiller, Friedrich

Schlegel, Friedrich

Schmidt, Arno

Schnitzler, Arthur

Scholem Alejchem

Schulz, Bruno

Schwitters, Kurt

Sciascia, Leonardo

Scorza, Manuel 
Scott, Walter

Seferis, Giorgios

Seghers, Anna

Sei Shōnagon

Seifert, Jaroslav

Sembène, Ousmane

Semprún, Jorge

Sender, Ramón José

Seneca

Senghor, Léopold Sédar

Sepehri, Sohräb

Seume, Johann Gottfried

Shakespeare, William

Shaw, George Bernard

Shaykh, Hanan al-

Shelley, Mary

Shelley, Percy Bysshe

Shepard, Sam

Shuihu zhuan $\rightarrow$ Räuber vom Liang Schan Moor

Sienkiewicz, Henryk

Sillitoe, Alan

Silone, Ignazio

Simenon, Georges

Simon, Claude

Sinclair, Upton

Singer, Isaac Bashevis

Sinjavskij, Andrej $\rightarrow$ Terc, Abram

Sinuhe-Roman

Sitwell, Edith

Sjöwall/Wahlöö

Skármeta, António

Skram, Bertha Amalie

Sobol, Joshua

Söderberg, Hjalmar

Södergran, Edith

Sollers, Philippe

Šolochov, Michail

Sologub, Fëdor

Solženicyn, Aleksandr

Sony Labou Tansi

Sophokles

Sorokin, Vladimir

Sow Fall, Aminata

Soyinka, Wole

Spark, Muriel

Spenser, Edmund

Staël, Madame de

Stagnelius, Erik Johan

Stein, Gertrude

Steinbeck, John

Stendhal

Sterne, Laurence
Sternheim, Carl

Stevens, Wallace

Stevenson, Robert Louis

Stifter, Adalbert

Stoker, Bram

Stoppard, Tom

Storm, Theodor

Stowe, Harriet Beecher

Strauß, Botho

Strindberg, August

Strittmatter, Erwin

Styron, William

Sue, Eugène

Svevo, Italo

Swift, Jonathan

Swinburne, Algernon Charles

Synge, John Millington

Szabó, Magda

Szczypiorski, Andrzej

Szymborska, Wisława

$\mathbf{T}$

Tabori, George

Tabucchi, Antonio

Tagore, Rabindranāth

Talmud

Tammsaare, Anton Hansen

Tanizaki, Jun'ichirō

Tasso, Torquato

Tausendundeiner Nacht, Die Erzählungen von

Tennyson, Alfred Lord

Terc, Abram

Terenz

Thackeray, William Makepeace

Thomas, Dylan

Thoreau, Henry David

Tibull

Tieck, Ludwig

Tirso de Molina

Tišma, Aleksandar

Tjutčev, Fedor

Toer, Pramudya Ananta

Tolkien, J.R.R.

Tolstaja, Tat'jana

Tolstoj, Lev

Tomasi di Lampedusa, Giuseppe

Tommaseo, Niccolò

Torga, Miguel

Tournier, Michel

Trakl, Georg

Tranströmer, Tomas

Traum der roten Kammer, Der 
Traven, $\mathrm{B}$.

Trifonov, Jurij

Tschechow, Anton $\rightarrow$ Cechov, Anton

Tucholsky, Kurt

Turgenev, Ivan

Tutuola, Amos

Twain, Mark $\rightarrow$ Mark Twain

U

Ugrešić, Dubravka

Unamuno, Miguel de

Under, Marie

Undset, Sigrid

Ungaretti, Giuseppe

Updike, John

\section{V}

Valera y Alcalá Galiano, Juan

Valéry, Paul

Vallejo, César

Vargas Llosa, Mario

Vazov, Ivan $\mathrm{M}$.

Veden

Vega, Lope de

Veli (Kanik), Orhan

Vera, Yvonne

Verga, Giovanni

Vergil

Veríssimo, Érico

Verlaine, Paul

Verne, Jules

Verwey, Albert

Vian, Boris

Vicente, Gil

Villon, François

Vittorini, Elio

Voltaire

Voznesenskij, Andrej

W

wa Thiong', Ngugi $\rightarrow$ Ngugi wa Thion's, James

Walcott, Derek

Walker, Alice

Wallace, Edgar

Walser, Martin

Walser, Robert

Waltari, Mika

Walther von der Vogelweide

Wang Anyi

Wang Meng

Waugh, Evelyn

Wedekind, Frank
Weiss, Peter

Wells, H.G.

Welty, Eudora

Werfel, Franz

Wergeland, Henrik

Wharton, Edith

White, Patrick

Whitman, Walt

Wieland, Christoph Martin

Wilde, Oscar

Wilder, Thornton

Williams, Tennessee

Williams, William Carlos

Wilson, Angus

Wolf, Christa

Wolfe, Thomas

Wolfram von Eschenbach

Wollstonecraft, Mary

Woolf, Virginia

Wordsworth, William

Wright, Richard

$\mathbf{Y}$

Yang Lian

Yeats, W.B.

Yoshimoto, Banana

Yourcenar, Marguerite

Yu Hua

$\mathbf{Z}$

Zamjatin, Evgenij

Zei, Alki

Zhang Ailing

Zola, Émile

Zorrilla y Moral, José

Zuckmayer, Carl

Zweig, Arnold

Zweig, Stefan

Zwetajewa, Marina $\rightarrow$ Cvetaeva, Marina 


\section{Mitarbeiterinnen und Mitarbeiter}

Ahrends, Günter: Christopher Marlowe

Ahrens, Rüdiger: John Osborne

Albert, Claudia: Heinrich Mann, Thomas Mann

Altenburger, Roland: Moruo Guo, (Die) Räuber vom Liang Schan Moor, (Der) Traum der roten Kammer

Ammon, Judith: Chen Ruoxi, Ding Ling

Anastasiadis, Athanasios: Aris Alexandrou, Dimitris Chatzis, Odysseas Elytis, Konstantinos Kavafis, Nikos Kazantzakis, Kostis Palamas, Jannis Ritsos, Emmanouil Roidis, Giorgios Seferis, Alki Zei

Angiolini, Alessia: Giorgio Bassani, Carlo Emilio Gadda, Giuseppe Tomasi di Lampedusa

Anz, Thomas: Friedrich Schlegel

Bach, Gerhard: Saul Bellow, Philip Roth, Isaac Bashevis Singer

Bachmaier, Helmut: Franz Grillparzer, Arthur Schnitzler, Arnold Zweig

Bajković, Dajana: Ivo Andrić, Ivan Cankar, Miroslav Krleža, Aleksandar Tišma, France Prešeren

Bauhaus-Lötzke, Hannelore: Premcand

Baumann, Uwe: Charlotte Brontë

Baumgart, Angelika: Guillaume Apollinaire, Antonin Artaud, André Breton, Michail Bulgakov, Jean Giraudoux, Eugène Ionesco, Alfred Jarry, Guy de Maupassant, Vladimir Nabokov, Raymond Queneau

Becheru, Sorina: Mircea Eliade, Mihai Eminescu Becker, Hans-Jürgen: Kabbala, Talmud

Berger, Dieter A.: Henry Fielding

Berkemeier, Christian: Paul Auster

Bernard, Wolfgang: Homer

Berndt, Katrin: Yvonne Vera

Berthold, Heinz: Mark Aurel

Beutin, Heidi: Marie von Ebner-Eschenbach

Beutin, Wolfgang: Sebastian Brant, Martin Luther, Fritz Reuter, Hans Sachs

Binder, Wolfgang: James Baldwin, V.S. Naipaul

Binias, Silke: Algernon Charles Swinburne

Bisanti, Tatiana: Pietro Aretino, Filippo Tommaso Marinetti, Eugenio Montale, Giovanni Pascoli, Giuseppe Ungaretti

Bischoff, Volker: Emily Dickinson, Robert Frost, Walt Whitman

Blamberger, Günter: Heinrich Böll, Adelbert von Chamisso
Blumenkamp, Katrin: Camilo José Cela, Jorge Semprún

Bobzin, Hartmut: Koran

Bohmeier, Ute: Samuel Josef Agnon, Jehuda Amichai, Aharon Appelfeld, Chaim Nachman Bialik, Abraham B. Jehoschua, Yoram Kaniuk, Amos $\mathrm{Oz}$, Joshua Sobol

Böldl, Klaus: Adam Oehlenschläger

Bollenbeck, Georg: Gottfried Keller, Conrad Ferdinand Meyer, Theodor Storm

Börchers, Sabine: Silvina Ocampo, Mercé Rodoreda

Borgmeier, Raimund: John Keats, W. Somerset Maugham, Samuel Richardson, William Wordsworth

Brandenberger, Tobias: Augustina Bessa-Luis

Brandt, Horst: Henri Alain-Fournier, Pierre Loti, Jacques Prévert

Braun, Hartmut: John Irving

Braungart, Wolfgang: Jeremias Gotthelf

Breinig, Helmbrecht: James Fenimore Cooper, Nathaniel Hawthorne, Washington Irving, Herman Melville, Edgar Allan Poe

Breitinger, Eckhard: Chinua Achebe, Ayi Kwei Armah, Syl Cheney-Coker, Dambudzo Marechera, Amos Tutuola

Breuer, Ingo: Heinrich von Kleist

Brillmann-Ede, Heike: Aminata Sow Fall

Bücher, Britta: Doris Lessing

Carbe, Monika: Sabahattin Ali, Fazll Hüsnü Dağlarca, Sait Faik, Nâzım Hikmet, Orhan Kemal, Yaşar Kemal, Aziz Nesin, Orhan Pamuk, Orhan Veli (Kanık)

Clausen, Bettina: Arno Schmidt

Coenen-Mennemeier, Brigitta: Marguerite Duras, Nathalie Sarraute

Collier, Gordon: Keri Hulme, David Malouf, Derek Walcott, Patrick White

Criegern de Guiñazú, Friederike von: Pablo Neruda

Cujai, Nicole: Nadine Gordimer, Katherine Mansfield

Davis, Geoffrey V.: Athol Fugard, Bessie Head

Detering, Klemens: Carlos Drummond de Andrade, Camilo Castelo Branco, Alexandre Herculano, João Guimarães Rosa, António Skármeta 
Dietschreit, Frank: Hans Magnus Enzensberger, Botho Strauß

Dietz, Ludwig: Georg Heym, Franz Kafka, Karl Krolow

Dittmann-Grönholm, Irja: Lydia Koidula, Viivi Luik

Diz Vidal, Martin: Jorge Guillén, Juan Ramón Jiménez

Döring-Smirnov, Johanna Renate: Anna Achmatova

Dörr, Gerhard: Claude Simon

Düring, Michael: Fazil' Iskander

Eckel, Winfried: Maurice Maeterlinck, Stéphane Mallarmé

Eglinger, Hanna: Per Olov Enquist, Peter Høeg

Ehlert, Klaus: Friedrich Schiller

Emmerich, Wolfgang: Erich Arendt, Johannes Bobrowski, Volker Braun, Paul Celan, Christoph Hein

Engel, Manfred: Rainer Maria Rilke

Ensslen, Klaus: Ralph Ellison, Toni Morrison, Alice Walker, Richard Wright

Ernst, Jutta: Joan Didion

Erzgräber, Willi: Geoffrey Chaucer, Thomas Hardy, Gerard Manley Hopkins

Eschweiler, Gabriele: Blaise Cendrars, Julio Cortázar, Manuel Puig, Ernesto Sábato, Mario Vargas Llosa

Feuchert, Sascha: Agatha Christie, Arthur Conan Doyle

Figueras, Mercedes: Max Aub

Fischer, Christine: Aleksandr Blok

Fischer, Katrin: Raymond Chandler, Dashiell Hammett

Fischer-Junghölter, Katrin: Jean Anouilh, Charles Baudelaire, François-René Chateaubriand, Victor Hugo, Marcel Proust, Charles-Augustin de Sainte-Beuve, Paul Verlaine

Fischer-Seidel, Therese: Samuel Beckett, E.M. Forster

Fluck, Winfried: Henry James, Mark Twain

Foltinek, Herbert: Charles Dickens

Fornaro, Sotera: Sappho

Franz, Norbert: Anton Čechov, Evgenij Zamjatin, Fedor Dostoevskij, Nikolaj Gogol', Nikolaj Leskov, Aleksandr Puškin

Freese, Peter: Bernard Malamud, J.D. Salinger

Freiburg, Rudolf: Samuel Johnson

Freudenthal, David: Ernesto Cardenal, Juan Carlos Onetti, Octavio Paz, Augusto Roa Bastos, Tirso de Molina

Friese, Wilhelm: Gunnar Gunnarsson, Jónas
Hallgrímsson, Halldór Laxness, Hallgrímur Pétursson

Frodl, Aglaja: Muriel Spark

Fuhrmann, Manfred: Seneca

Gago, Carla: Jorge Amado, Joaquim Maria Machado de Assis

Gall, Dorothea: Catull

Gebhardt, Lisette: Murasaki Shikibu

Gehring, Wolfgang: Alan Sillitoe

Gelfort, Claus: Botho Strauß

Glaap, Albert-Reiner: Alan Ayckbourn

Glomb, Stefan: Tom Stoppard

Göbler, Frank: Evgenij Baratynskij, Ivan Bunin, Daniil Charms, Vladislav Chodasevič, Gavrila Deržavin, Ivan Gončarov, Nikolaj Karamzin, Michail Lermontov, Michail Lomonosov, Aleksandr Ostrovskij, Fedor Tjutčev, Lev Tolstoj, Ivan Turgenev

Götsch, Dietmar: Carl Jonas Love Almqvist, Hans Christian Andersen, Carl Michael Bellman, Hjalmar Söderberg, Erik Johan Stagnelius, Tomas Tranströmer

Graeber, Wilhelm: Pierre Augustin Caron de Beaumarchais, Dino Buzzati, Italo Calvino, Federico García Lorca, François de La Rochefoucauld, Niccolò Machiavelli, Pierre Carlet de Marivaux, François Mauriac, Molière, Italo Svevo, Antonio Tabucchi, Giovanni Verga

Grandel, Hartmut: Langston Hughes

Grewe-Volpp, Christa: Robert Creeley

Grimm, Gunter E.: Stefan George, Paul Heyse, Franz Werfel

Gröne, Maximilian: Georges Bernanos, Michel Butor, Baldassare Castiglione, Alphonse Daudet, Jean Giono, Joris-Karl Huysmans, Roger Martin du Gard, Henry de Montherlant, Alberto Moravia, Alfred de Musset

Guthke, Karl S.: B. Traven

Györffy, Miklós: Endre Ady, Tibor Déry, Péter Esterházy, Attila József, Imre Kertész, Dezső Kosztolányi, Iván Mándy, Sándor Márai, Miklós Mészöly, Ferenc Molnár, Péter Nádas, László Németh, Sándor Petőfi

Haas, Alexandra: Caryl Phillips

Habermehl, Peter: Augustinus, Petron

Habersetzer, Karl-Heinz: Clemens Brentano, Heimito von Doderer, Hugo von Hofmannsthal, Johann Nepomuk Nestroy

Hallof, Klaus u. Luise: Hesiod

Harich-Schwarzbauer, Henriette: Alexanderroman Harreß, Birgit: Hanna Krall 
Hartman, Michelle: Gāda as-Sammān

Hartwig, Sebastian: René Char, Émile Michel Cioran, Paul Claudel, Jean Cocteau, Paul Éluard, Don Luis de Góngora y Argote, Antonio Machado y Ruiz, Gérard de Nerval, Jean Arthur Rimbaud, Saint-John Perse

Hasselblatt, Cornelius: Jaan Kross, Anton Hansen Tammsaare, Marie Under

Hauschild, Jan-Christoph: Georg Büchner, Georg Herwegh

Havemann, Miriam: Roland Barthes, Jorge Luis Borges, Albert Camus, Pierre Corneille, Jean Genet, André Gide, Marcel Pagnol, Georges Perec, Jean Racine, Alain Robbe-Grillet

Hebel, Udo: William Bradford

Heide, Markus: Paul Bowles

Heidtmann, Horst: Achim von Arnim, Joseph von Eichendorff

Heitmann, Annegret: Johannes Vilhelm Jensen, Alexander Lange Kielland, Sigrid Undset

Henke, Florian: Yves Bonnefoy, Chrestien de Troyes, Pierre Drieu la Rochelle, Julien Gracq, Jean de La Fontaine, Giacomo Leopardi, Cesare Pavese, Francesco Petrarca, François Rabelais, Michel Tournier

Herget, Winfried: William Cullen Bryant Herlth, Jens: Iosif Brodskij, Osip Mandelštam Hijiya-Kirschnereit, Irmela: Abe Kōbō, Akutagawa Ryūnosuke, Bashō, Enchi Fumiko, Endō Shusaku, Inoue Yasushi, Kawabata Yasunari, Köno Taeko, Mishima Yukio, Mori Ōgai, Murakami Haruki, Natsume Sōseki, Ōe Kenzaburō, Sei Shōnagon, Tanizaki Jun'ichirō

Hilf, Susanne: Romesh Gunesekera, Michael Ondaatje

Hilzinger, Sonja: Gertrud Kolmar, Anna Seghers

Himberg, Kay: Lawrence Durrell

Hof, Renate: Joyce Carol Oates

Höfele, Andreas: William Shakespeare, Oscar Wilde

Hoff, Karin: Karin Boye

Hofstra, Diethelm: Mochtar Lubis, Rendra, Pramudya Ananta Toer

Hölbling, Walter: Ernest Hemingway

Hollweg, Brenda: Stephen King

Holzberg, Niklas: Ovid

Hopfe, Karin: Gabriela Mistral

Horatschek, Anna-M.: Peter Ackroyd

Horlacher, Stefan: John Fowles

Horst, Simone: Edda

Horster, Detlef: Aristoteles

Horstmann, Ulrich: Ted Hughes, Philip Larkin
Hotz, Karl: Siegfried Lenz, Nelly Sachs, Adalbert Stifter, Erwin Strittmatter

Hoven, Heribert: Malcolm Lowry

Hristozova, Miglena: Boris Pasternak, Vladimir Sorokin, Ivan M. Vazov

Huber, Werner: Brendan Behan, Sean O'Casey

Hühn, Peter: John le Carré

Humphrey, Richard: John Arden, John Galsworthy

Hurm, Gerd: Djuna Barnes, John Dos Passos, F. Scott Fitzgerald, Richard Ford, Gertrude Stein

Ickstadt, Heinz: William Gaddis, Thomas Pynchon

Igler, Susanne: Antonio Muñoz Molina, Romain Rolland

Imhof, Rüdiger: John Banville

Jansen, Hans: Robert Musil

Jarfe, Günther: W.H. Auden

Jekutsch, Ulrike: Isaak Babel', Velemir Chlebnikov

Jolles, Charlotte: Theodor Fontane

Joost, Ulrich: Karl Kraus

Jorgensen, Bo Hakon: Karen Blixen

Kalb, Kristina: Elizabeth Bishop

Karge, Gesine: Thomas Mann

Karimi, Kian-Harald: Adolfo Bioy Casares, Clarín, Alexandre Dumas (père), Juan Valera y Alcalá Galiano

Karrer, Wolfgang: Dylan Thomas

Kaukoreit, Volker: Erich Fried

Keil-Sagawe, Regina: Assia Djebar, Nawāl asSa’adāwī

Keitel, Evelyne: Patricia Highsmith

Kelleter, Frank: Henry Miller

Kessler, Stephan: Amanda Aizpuriete, Nora

Ikstena, Jurga Ivanauskaitė, Jurgis Kunčinas

Klein, Holger: J.B. Priestley

Kleinert, Susanne: Dacia Maraini, Elsa Morante

Klepper, Martin: Richard Brautigan

Knopf, Jan: Bertolt Brecht, Friedrich Dürrenmatt, Georg Trakl

Köbke, Jörg: Reinaldo Arenas, José Lezama Lima

Kohl, Stephan: William Makepeace Thackeray

Köhnen, Ralph: Herta Müller

König, Alexandra: Colette

Korte, Barbara: H.G. Wells

Kramer, Jürgen: Joseph Conrad

Krapinger, Gernot: Sallust

Krapoth, Hermann: Benjamin Constant

Kravec, Viktor: Vladimir Majakovskij

Kreidt, Dietrich: Ödön von Horváth

Kretschmer, Ernst: H.C. Artmann, Christian Morgenstern, Joachim Ringelnatz

Kreutzer, Eberhard: Lewis Carroll, Amitav Ghosh, James Joyce, Salman Rushdie, W.B. Yeats 
Krewani, Angela: Angela Carter

Krings, Constanze: Jean Rhys

Kroll, Renate: Simone de Beauvoir

Kröner, Hans-Otto: Cicero, Tibull, Vergil

Kubin, Wolfgang: Bei Dao, (Das) Buch der Lieder, (Das) Buch der Wandlungen, Du Fu, Yang Lian

Kulessa, Rotraud von: Elio Vittorini

Kullmann, Thomas: Emily Brontë, Edmund Spenser, Alfred Lord Tennyson

Küppers, Gabriele: Gioconda Belli

La Salvia, Adrian: Dante Alighieri

Lange, Bernd-Peter: George Orwell

Lange, Ulrike: Abram Terc

Lasch, Markus: Augusto Boal, João Cabral de Melo Neto, António Ferreira, Francisco Manuel de Melo

Lehmann, Jessica: Andrej Belyj, Aleksej Remizov

Leitner, Claudia: Isabel Allende

Lengeler, Rainer: Robert Browning

Lersch-Schumacher, Barbara: Anna Blaman, Louis Paul Boon, Hugo Claus, Desiderius, Erasmus von Rotterdam, Guido Gezelle, Hella S.

Haasse, Maarten 't Hart, A.F.Th. van der Heijden, Willem Frederik Hermans, Judith Herzberg, Pieter Corneliszoon Hooft, Willem Johannes Theodorus Kloos, Lucebert, Margriet de Moor, Harry Mulisch, Multatuli, Cees Nooteboom, Monika van Paemel, Gerard Reve, Albert Verwey

Lessenich, Rolf: William Blake, Lord Byron, Daniel Defoe, John Dryden, Oliver Goldsmith, John Milton, Mary Shelley

Leypoldt, Günter: William Faulkner, Jack Kerouac Liebel, Helmuth: Wilhelm Raabe

Lindemann, Uwe: Nicolas Boileau, Pierre Ambroise François Choderlos de Laclos, Michel Houellebecq, Antoine de SaintExupéry, Leonardo Sciascia

Link, Viktor: Samuel Taylor Coleridge, John Millington Synge

Lohse, Rolf: Pierre Abélard, Fernando Arrabal, Guillermo Cabrera Infante, Gustave Flaubert, Ramón Gómez de la Serna, Edmond u. Jules de Goncourt, Pierre Mertens, Eugène Sue, Niccolò Tommaseo, Émile Zola

Loimeier, Manfred: Amadou Hampâté Bâ, Tahar Ben Jelloun, Mongo Beti, Rachid Boudjedra, Mia Couto, Bernard B. Dadié, Mohammed Dib, Abdulrazak Gurnah, Yacine Kateb, Ahmadou Kourouma, Albert Memmi, V.Y. Mudimbe, Meja Mwangi, Patrice Nganang,
Yambo Ouologuem, Pepetela, Ousmane Sembène, Léopold Sédar Senghor, Sony Labou Tansi

Löschnigg, Maria: Edward Bond

Lutz, Bernd: Alfred Andersch, Ingeborg Bachmann, Hans Fallada, Karl May

Lutz, Hartmut: Louise Erdrich

Mabana, Kahiudi Claver: Aimé Césaire

Mader, Doris: Caryl Churchill

Mai, Anne-Marie: Inger Christensen

Martens, Ekkehard: Platon

Mathis, Ursula: Anne Hébert

Matzke, Monika: Forūg Farroḩzād

Maul, Stefan M.: Gilgamesch-Epos

Meid, Volker: Hans Jacob Christoph von Grimmelshausen, Andreas Gryphius, Daniel Caspar von Lohenstein

Meinig, Sigrun: Peter Carey

Mende, Dirk: Erich Maria Remarque

Mertin, Ray-Güde: Clarice Lispector

Meyer, Michael: Buchi Emecheta

Meyer-Krentler, Leonie: Roberto Bolaño, Rafael Chirbes, Javier Marías

Middeke, Martin: Samuel Butler, Thomas Carlyle, Bram Stoker

Modick, Klaus: Lion Feuchtwanger, Ernst Jünger

Moers, Gerald: Sinuhe-Roman

Moraldo, Sandro: Dario Fo, Carlo Goldoni, Luigi Pirandello

Moss, Maria: John Barth, Don DeLillo

Moster, Stefan: Minna Canth

Mrugalla, Georg: Jerzy Andrzejewski, Kazimierz Brandys, Witold Gombrowicz, Zbigniew Herbert, Marek Hłasko, Jan Kochanowski, Tadeusz Konwicki, Andrzej Kuśniewicz, Stanisław Jerzy Lec, Stanisław Lem, Adam Mickiewicz, Czesław Miłoz, Sławomir Mrożek, Maria Nurowska, Tadeusz Rózewicz, Bruno Schulz, Henryk Sienkiewicz, Andrzej Szczypiorski

Müllenbrock, Heinz-Joachim: Alexander Pope, Walter Scott

Müller, Klaus Peter: Harold Pinter

Müller, Kurt: Arthur Miller, Eugene O'Neill

Müller, Wolfgang G.: Jane Austen, Robert Burns,

Truman Capote, Raymond Carver, E.E. Cummings, John Donne, T.S. Eliot, Thomas Gray, George Herbert, Ben Jonson, Ezra Pound, Percy Bysshe Shelley, Wallace Stevens, John Updike, William Carlos Williams Müller, Wolfgang: Hans Joachim Schädlich Müller-Hanpft, Susanne: Günter Eich 
Müller-Wood, Anja: Sarah Kane, Thomas Middleton

Müller-Zettelmann, Eva: Edith Sitwell

Munro, Irmtraut: Altägyptisches Totenbuch

Naguschewski, Stephan: Beowulf, Carmina Burana, Hildebrandslied

Naumann, Ursula: Karoline von Günderrode

Naumann, Uwe: Klaus Mann

Nesselrath, Heinz-Günther: Lukian

Neuhaus, Stefan: Martin Walser

Neuhaus, Volker: Günter Grass

Niederhoff, Burkhard: William Congreve, Robert Louis Stevenson

Nieragden, Göran: Kingsley Amis

Nilsson, Gunnar: Juan Benet

Nischik, Reingard M.: Margaret Atwood, Alice Munro

Nix, Angelika: Selma Lagerlöf

Nover, Peter: Anthony Burgess

Nünlist, René: Archilochos

Nünning, Ansgar: Sherwood Anderson, Aphra Behn

Nünning, Vera: Aphra Behn, Mary Wollstonecraft

Oesterheld, Christina: Mirzā Asadullāh Kֵhān Gālib

Ohde, Horst: Helmut Heißenbüttel, Ernst Jandl, Kurt Schwitters

Opfermann, Susanne: Harriet Beecher Stowe

Ophüls-Kashima, Reinold: Yoshimoto Banana

Opitz, Michael: Durs Grünbein, Elfriede Jelinek, Heiner Müller

Opitz-Wiemers, Carola: Christine Lavant

Ott, Claudia: Koran, Múallaqāt

Paatz, Annette: Juan Goytisolo, Benito Pérez Galdós, Fernando de Rojas

Parnell, Christina: Ljudmila Petruševskaja, Tat'jana Tolstaja

Pehnt, Annette: John Steinbeck

Peters, Christoph M.: Aldous Huxley

Petersen, Jürgen H.: Max Frisch

Petzold, Dieter: Enid Blyton

Pfeiffer, Erna: Sor Juana Inés de la Cruz, Rosario Ferré, Cristina Peri Rossi

Pflitsch, Andreas: Adonis, Rashid al-Daif, Mahmud Darwish, Gamal al-Ghitani, Khalil Gibran, Emil Habibi, Ghassan Kanafani, Edwar al-Kharrat, Elias Khoury, Ibrahim al-Koni, Amin Maalouf, Nagib Machfus, Abdalrahman Munif, Tayyib Salih, Hanan al-Shaykh, Tausendundeine Nacht

Pix, Gunther: Robert Walser
Pohl, Burkhard: Andrés Bello, Alejo Carpentier, Miguel de Cervantes

Pohl, Christian: Rolf Hochhuth

Pollmann, Karla: Anakreon

Porsche, Michael: E.L. Doctorow

Pressler, Frank: Antimachos von Kolophon

Prießnitz, Horst: Les Murray

Pron, Patricio: Vicente Blasco Ibáñez, Cantar de Mío Cid, Lope de Vega

Raabe, Paul: Else Lasker-Schüler

Rakusa, Ilma: Marina Cvetaeva

Rauße, Astrid: Maryse Condé

Real, Hermann J.: Jonathan Swift

Reckwitz, Erhard: Breyten Breytenbach, André Brink, J.M. Coetzee, Iris Murdoch

Rehder, Petra: Dubravka Ugrešić

Rehm, Ortrun: Kerstin Ekman, Sara Lidman, Henning Mankell, Sjöwall/ Wahlöö

Reichardt, Dagmar: Aimé Césaire

Reichardt, Ulfried: John Ashbery, Sylvia Plath

Reinhart, Werner: Edward Albee, Tennessee Williams

Reiser, Frank: Pío Baroja y Nessi, Gustavo Adolfo Bécquer, Pedro Calderón de la Barca, Gracián, José Ortega y Gasset, Luis Cernuda, Rubén Dario, Alexandre Dumas (fils), Ramón José Sender, José Zorrilla y Moral

Reitz, Christiane: Lukrez

Riedel, Annette: Grazia Deledda, Natalia Ginzburg, Anna Maria Ortese

Riedel, Nicolai: Uwe Johnson, Günter Kunert

Riemann, Wolfgang: Diwan-Poesie

Riemenschneider, Dieter: Mulk Raj Anand, Anita Desai, R.K. Narayan, Raja Rao, Arundhati Roy

Riemenschnitter, Andrea: Gao Xingjian, Mo Yan, Wang Anyi, Zhang Ailing

Rippl, Gabriele: A.S. Byatt

Roberg, Thomas: Novalis

Röseberg, Dorothee: Agota Kristof

Rössler, Andrea: Rosa Montero

Rothmeier, Christa: Věra Linhartová, Božena Němcová

Ruckaberle, Axel: Jean Cayrol, Julien Green

Rühling, Lutz: Edith Södergran

Saalfeld, Lerke von: August Heinrich Hoffmann von Fallersleben

Sabin, Stefana: Tudor Arghezi, Ludovico Ariost, Honoré de Balzac, Giovanni Boccaccio, Michelangelo Buonarotti, Giovanni Giacomo Casanova, Primo Levi, André Malraux, Frédéric Mistral, Jean-Paul Sartre

Sagaster, Börte: Adalet Ağaoğlu 
Salheiser, Britta: Stephen Crane

Sander, Hans Jochen: William Golding, Angus Wilson

Sander, Ulrike-Christine: Herman Bang, Gunnar Ekelöf, Lars Gustafsson, Knut Hamsun, Ludvig Holberg, Henrik Ibsen, Jens Peter Jacobsen, Søren Kierkegaard, Bjarne Reuter, August Strindberg

Sareika, Rüdiger: Francisco Sionil José

Sarrey-Strack, Colette: Annie Ernaux, Marguerite Yourcenar

Saynovits, Ilse: Ana Blandiana

Schäffner, Raimund: George Bernard Shaw

Schafroth, Heinz F.: Ilse Aichinger

Scharf, Kurt: Bozorg Alavi, 'Ațțār, Luís Vaz de Camões, Omar Chajjām, Maḥmud Doulatābādi, Moulana Nuroddin Abdorrahman Dschāmi, José Maria Eça de Queirós, Ferdousi, José Rubem Fonseca, João Baptista da Silva Garrett, Hušang Golširi, Faḩrơd-din Aśad Gorgāni, Hafis, Saādeq Hedāyat, António Lobo Antunes, Abbas Maroufi, Abu Mohammad Nisami, Fernando Pessoa, Rumi, Saadi, José Saramago, Ahmad Schämlu, Sohrab Sepehri, Miguel Torga, Érico Veríssimo

Scharold, Irmgard: Tommaso Landolfi, J.M.G. Le Clézio, Prosper Mérimée, Pier Paolo Pasolini

Scheffel, Gerda: Robert Pinget

Schenkel, Elmar: Bruce Chatwin

Scheuer, Helmut: Gerhart Hauptmann, Stefan Zweig

Schiedermair, Joachim: Pär Lagerkvist, Henrik Wergeland

Schlaeger, Jürgen: William Beckford, Seamus Heaney, Ian McEwan, Samuel Pepys

Schlaffer, Hannelore: Johann Wolfgang Goethe

Schlüter, Gisela: Giovanni Battista Guarini, Charles-Louis de Montesquieu, Jean-Jacques Rousseau, Stendhal

Schmidt, Klaus Elmar: Miguel Ángel Asturias Schmidt, Siegfried J.: Friederike Mayröcker Schmidt-Welle, Friedhelm: Carlos Fuentes, Julio Llamazares, Juan Rulfo, Manuel Scorza, César Vallejo

Schmitz, Matthias: Thomas Bernhard

Schmitz-Köster, Dorothee: Sarah Kirsch, Irmtraud Morgner

Schneider, Ronald: Annette von Droste-Hülshoff

Schneider, Thomas: Christian Dietrich Grabbe

Schnitker, Jan: Francis Bacon

Scholz, Anne-Marie: Edith Wharton

Scholz, Ute: Fëdor Abramov, Boris Pil'njak
Schöneich, Christoph: Graham Greene

Schönert, Jörg: Carl Sternheim, Frank Wedekind

Schöpp, Joseph C.: Donald Barthelme, William

Burroughs, Robert Coover, Raymond

Federman

Schrey-Vasara, Gabriele: Bo Carpelan, Paavo Haavikko, Aleksis Kivi, Eino Leino, Rosa Liksom, Väinö Linna, Elias Lönnrot, Veijo Meri, Pentti Saarikoski, Mika Waltari

Schuerkens, Ulrike: Mariama Bâ

Schulz, Dieter: Ralph Waldo Emerson, Henry David Thoreau

Schulz, Genia: Marieluise Fleißer, Heiner Müller, Peter Weiss, Christa Wolf

Schulze-Engler, Frank: James Ngugi wa Thiongo, Ken Saro-Wiwa, Wole Soyinka

Schütte, Wolfram: Hubert Fichte

Schütte-Weißenborn, Birgit: Elfriede Jelinek

Schwab, Hans-Rüdiger: Bettine von Arnim, Jakob u. Wilhelm Grimm, Wilhelm Hauff, Johann Peter Hebel, Luise Rinser

Schweiger, Irmy: Yu Hua

Schweikert, Uwe: Elias Canetti, Alfred Döblin, Hans Henny Jahnn, Jean Paul, Marie Luise Kaschnitz, Brigitte Kronauer, Ludwig Tieck

Schweikle, Günther: Gottfried von Straßburg, Hartmann von Aue, Neidhart, Nibelungenlied, Walther von der Vogelweide, Wolfram von Eschenbach

Seeber, Hans Ulrich: George Eliot

Seibel, Klaudia: J.K. Rowling, J.R.R Tolkien

Sherberg, Barbara: Menander

Sichert, Margit: Sam Shepard

Sielke, Sabine: Rita Dove

Simon, Hans-Ulrich: Eduard Mörike

Simonis, Annette: Gabriele D'Annunzio, Théophile Gautier, Henri Michaux, Blaise Pascal, Torquato Tasso

Simonis, Linda: Francisco de Quevedo, Miguel de Unamuno, Denis Diderot, Paul Valéry

Sitzler, Kathrin: Magda Szabó

Smolka, Andrea-Eva: José Sobral de Almada

Negreiros, Lídia Jorge, Gil Vicente

Späth, Eberhard: Edgar Wallace

Spies, Marion: Janet Frame

Stark, Michael: Gottfried Benn

Steinfeld, Torill: Bertha Amalie Skram

Steltner, Ulrich: Il'ja Ehrenburg, Andrej Voznesenskij

Stephan, Inge: Johann Gottfried Seume

Stratmann, Gerd: Laurence Sterne

Strümpel, Jan: George Tabori 
Stummer, Peter: Nuruddin Farah

Surkamp, Carola: Thornton Wilder

Surmatz, Astrid: Astrid Lindgren

Syndikus, Hans Peter: Horaz

Thiele, Eckhard: Evgenij Evtušenko, Fedor

Sologub, Jurij Trifonov

Thielmann, Pia: Ama Ata Aidoo

Thurner, Bettina: Thomas Wolfe

Tonn, Horst: Norman Mailer

Trappl, Richard: Wang Meng

Tscherpel, Roland: Christian Friedrich Hebbel,

Erich Kästner

Unterweg, Friedrich-K.: Evelyn Waugh

Vaßen, Florian: Rolf Dieter Brinkmann, Wilhelm Busch, Heinrich Heine

Vester, Heinz-Günter: Umberto Eco, Gabriel Garcia Márquez

Virkus, Fred: Kalidasa, Kamasutra, Veden

Vöhler, Martin: Pindar

Vollhardt, Friedrich: Hermann Broch, Gotthold Ephraim Lessing

Voulgari, Sophia: Aris Alexandrou, Dimitris Chatzis, Odysseas Elytis, Konstantinos Kavafis, Nikos Kazantzakis, Kostis Palamas, Jannis Ritsos, Emmanouil Roidis, Giorgios Seferis, Alki Zei

Vykoupil, Susanna: Karel Čapek, Jaroslav Hašek, Václav Havel, Bohumil Hrabal, Klára Jarunková, Peter Karvaš, Ivan Klíma, Pavel Kohout, Jiří Koláŕ, Milan Kundera, Ladislav Mnacko, Jan Neruda, Jaroslav Seifert

Wackwitz, Stephan: Friedrich Hölderlin, Hermann Lenz, Karl Philipp Moritz

Wagner-Egelhaaf, Martina: Peter Handke

Waligora, Melitta: Bhagavadgitā, Mahābhārata, Rāmāyana

Walter, Klaus-Peter: Čingiz Ajtmatov, Vasilij Aksënov, Andrej Bitov, Milovan Djilas, Maksim Gor'kij, Daniil Granin, Ismail Kadare, Konstantin Paustovskij, Viktor Pelevin, Andrej Platonov, Anatolij Rybakov, Scholem Alejchem, Georges Simenon, Michail Šolochov, Aleksandr Solženicyn

Walther, Wiebke: Abū l-Farağ al-Ișfahānī, Abū Muhammad al-Harîīi, Ibn al-'Arabì, 'Alì Ibn Hazm, Abü l-'Aläà al-Ma’arrī

Weber, Lea: Eyvind Johnson

Weglage, Matthias: Aesop

Wehdeking, Volker: Hermann Hesse, Wolfgang Koeppen, Carl Zuckmayer

Wehinger, Brunhilde: Hélène Cixous, George Sand, Madame de Staël
Wehnert, Jürgen: Bibel

Wehrheim, Monika: José Joaquín Fernández de Lizardi

Weigelin-Schwiedrzik, Susanne: Ai Qing, Ba Jin, Lao She, Lu Xun

Weiß, Christian: Mahasweta Devi, Rabīndranāth Tagore

Weiß, Michaela: Louis Aragon, Georges Bataille, Louis-Ferdinand Céline, Anatole France, Romain Gary, Edouard Glissant, Michel de Montaigne, Donatien Alphonse François Marquis de Sade, Philippe Sollers, Boris Vian, François Villon

Welz, Stefan: John Berger, Rudyard Kipling

Werner, Florian: Allen Ginsberg

Westermann, Klaus: Joseph Roth

Weyergraf, Bernd: Kurt Tucholsky

Wichner, Ernest: Mircea Dinescu, Gellu Naum Williams, Rhys W.: Elisabeth Langgässer

Wilsch, Kerstin: Sahar Khalifa

Wimmer, Susanne: Walter Kempowski

Winkgens, Meinhard: D.H. Lawrence

Winter, Hans-Gerd: Johann Gottfried Herder, Jakob Michael Reinhold Lenz, Peter Rühmkorf

Witt, Nicole: Juan Marsé, Miguel Delibes

Witt, Sabine: Massimo Bontempelli, Carlo Levi, Curzio Malaparte, Alessandro Manzoni, Ignazio Silone, Jules Verne

Wöbcke, Rita: (La) Chanson de Roland, Michel Leiris, Pierre de Ronsard, Voltaire

Woldan, Alois: Wisława Szymborska

Wolf, Werner: Virginia Woolf

Wolf-Grieshaber, Katharina: Danilo Kiš, Bora Ćosić

Yngborn, Katarina: Martin Andersen Nexø, Tove Jansson

Zacharasiewicz, Waldemar: Carson McCullers, Flannery O'Connor, William Styron, Eudora Welty

Zacher, Klaus-Dieter: Lukrez

Zemmrich, Katrin: Vera Mutafčieva

Zerweck, Bruno: Martin Amis

Zimmermann, Bernhard: Aischylos, Aristophanes, Euripides, E.T.A. Hoffmann, Plautus, Sophokles, Terenz, Christoph Martin Wieland

Zimmermann, Harro: Friedrich Gottlieb Klopstock

Zimmermann, Jutta: Jack London, Upton Sinclair Zimmermann, Margarete: Christine de Pizan 